\title{
A Rindler-KAM Spacetime Geometry and Scaling the Planck Scale Solves Quantum Relativity and Explains Dark Energy
}

\author{
Mohamed S. El Naschie \\ Department of Physics, University of Alexandria, Alexandria, Egypt \\ Email: Chaossf@aol.com
}

Received October 8, 2013; revised November 6, 2013; accepted November 14, 2013

Copyright (C) 2013 Mohamed S. El Naschie. This is an open access article distributed under the Creative Commons Attribution License, which permits unrestricted use, distribution, and reproduction in any medium, provided the original work is properly cited.

\begin{abstract}
We introduce an ultra high energy combined KAM-Rindler fractal spacetime quantum manifold, which increasingly resembles Einstein's smooth relativity spacetime, with decreasing energy. That way we derive an effective quantum gravity energy-mass relation and compute a dark energy density in complete agreement with all cosmological measurements, specifically WMAP and type 1a supernova. In particular we find that ordinary measurable energy density is given by $E_{1}=m c^{2} / 22$ while the dark energy density of the vacuum is given by $E_{2}=m c^{2}(21 / 22)$. The sum of both energies is equal to Einstein's energy $E=m c^{2}$. We conclude that $E=m c^{2}$ makes no distinction between ordinary energy and dark energy. More generally we conclude that the geometry and topology of quantum entanglement create our classical spacetime and glue it together and conversely quantum entanglement is the logical consequence of KAM theorem and zero measure topology of quantum spacetime. Furthermore we show via our version of a Rindler hyperbolic spacetime that Hawking negative vacuum energy, Unruh temperature and dark energy are different sides of the same medal.
\end{abstract}

Keywords: Quantum Relativity; KAM Theorem; Dark Energy; Hawking Negative Energy Vacuum Fluctuation; Unruh Temperature; Rindler Spacetime; Einstein-Rosen Bridges; Action at Distance; Susslin Operation

\section{Introduction}

A Kolmogorov-Arnold-Moser (i.e. KAM) Cantorian spacetime manifold rather than a simple fractal is the right starting point for an exact formulation of a quantumrelativity gravity theory which resolves the challenging task of explaining the increased rather than decreased rate of cosmic expansion and calculating accurately the measured $95.5 \%$ missing energy density of the universe [1-5]. Within the KAM formulation the thin fractal, i.e. zero measure random Cantor set is modelled by the zero set which is found from K-category theory to be given by the bi-dimension $D(0)=(0, \phi)$ and identified physically with a virtual quantum pre-particle [3-28]. On the other hand the fat fractal, i.e. the positive measure empty random Cantor set is given by $D(-1)=\left(-1, \phi^{2}\right)$ and is identified with a virtual pre-quantum wave being the cobordism of the quantum pre-particle zero set and $\phi=2 /(\sqrt{5}+1)$ [2-28]. Continuing the K-theoretical analysis [11-22] to encompass Penrose's noncommuta- tive fractal tiling which constitutes a compactified Klein modular holographic boundary for our KAM spacetime bulk $[8,9]$, we can define mass as the inverse of isomorphic diameters of the relevant spaces, for instance $2 \rho=4+\phi^{3}$ of Penrose tiling $[11,16,19]$. Simple analogies with positive and negative Van der Waals fluctuation, instantons dynamics and Hawking's negative energy vacuum fluctuation as well as Rindler coordinates at a toy black hole horizon lead us to understand negative gravity and dark energy [29-59]. Our formal analysis starts with an inspiring idea due to D. Gross [1] and arrives that way at a topological Planck energy $E_{p}$ and a corresponding topological Planck length $\ell_{P}$ effectively scaling the Planck scale from esoterically large $E_{p} \simeq 10^{19} \mathrm{Gev}$ and equally esoterically small $\ell_{P} \simeq 10^{-33}$ $\mathrm{cm}$ numbers to a manageably

$E_{P}=1 / \ell_{P}=P(H)=\phi^{5}=11+\phi^{5}$ where $P(H)$ is the famous Hardy's probability for quantum entanglement which amounts to almost 9 percent and $\phi=2 /(1+\sqrt{5})$ [3]. Based on these results we conclude the equivalence 
of Einstein-Rosen “wormhole" bridges and Einstein's Podolsky-Rosen's spooky action at distance [21]. In turn these results are shown to be consistent with distinguishing two energy components which result in Einstein's famous formula $E=m c^{2}$, namely the quantum zero set particle component $E(0)=m c^{2} / 22$ which we can measure and the quantum empty set wave component which due to state vector reduction on measurement we cannot measure $E(D)=m c^{2}(21 / 22)$, i.e. the missing dark energy [24-28]. Together the two components add to $E=E(0)+E(D)=E($ Einstein $)=m c^{2}$ where $E$ is the total energy, $m$ is the mass and $c$ is the speed of light [2-61]. In other words, the present new derivation of the world's most celebrated formula explains in one stroke the two most puzzling problems of quantum physics and relativistic cosmology, namely the physico-mathematical meaning of the wave function and the nature of dark energy [30-68]. In essence they are one and the same when looked upon from the view point of quantum-fractal geometry and KAM theorem [2,7,19,69-83].

\section{Motivation and Background Information}

The theory advocated in this paper is a synthesis of several fundamental ideas. The first idea is the recognition that using the KAM manifold well known and quite familiar from the theory of nonlinear dynamical systems and quantum chaos $[8,80]$ as the geometry of our quantum spacetime is by far a mathematically more firm and solid ground to build our physics upon than ordinary continuous fractal spacetime [81]. The second idea is that of scaling the Planck scale and working with topological quantities. In a remarkable essay [1] the co-founding father of Heterotic superstring theory and strong interaction asymptotic freedom, D. Gross gave a novel general direction for the unification of all fundamental forces and arriving at quantum gravity via an imaginative, unconventional and in fact revolutionary idea of scaling the almost esoteric Planck scale [1,2]. In the present work which tackles one of the currently most researched and hotly debated problems in quantum physics and cosmology [1-72], we take this idea literally and seriously to mean a mathematical topological formulation of physics starting from a unit interval spacetime with non-classical and non-smooth transfinite discrete geometry and topology [2-5]. Proceeding in this way we arrive at a quintessential, i.e. five dimensional Kaluza-Klein spacetime $[26,30]$ and a general formula for quantum entanglement $P=\phi^{n+3}$ which reduces in the case of two particles $n=2$ to Hardy's famous probability [16-21] of quantum entanglement $P(H)=\phi^{5}$ where $\phi=2 /(1+\sqrt{5})$, i.e. a quantum probability of almost $9 \%$. This result was repeatedly verified experimentally with a very high degree of accuracy $[3,4]$.
In the present work we show how $P(H)=\phi^{5}$ is related to Gross' proposal $[1,2]$ and discusses its various ramifications for dark energy [2,3] and the connection between general relativity's Einstein-Rosen bridges [24, 25 ] and the quantum mechanics of Einstein-PodolskyRosen nonlocality $[3,4,6]$. This insight leads to an effective quantum gravity theory $[69,70]$ and an explanation for the true meaning of the quantum wave function and the missing dark energy of the cosmos by fusing Einstein's relativity and quantum mechanics entanglement together $[3,4]$. Our basic philosophy and technical strategy may be summed up succinctly as follows: By facing infinity and zero head on and endorsing them we eliminate the drawback of both and suddenly everything in physics looks right [44-54]. Although the present work is reasonably self contained, for a deep understanding of the transfinite nonlinear techniques used to develop our theory it is helpful to read carefully at least Refs. [1,3,5] as well as Refs. [58-72] for a bird's eye view of the general theory $[5,16]$. We may also note that the theory of quantum sets was extensively developed by D. Finkelstein who also introduced the notion of quantum relativity [69-77]. We should add at this point that the KAM Cantorian spacetime theory has wider ramifications than only physics and cosmology and has found applications in brain and consciousness research by M. Persinger, C. Lavalle [78] as well as S. Vrobel's fundamental discourses into time, perception, music and love [79].

\section{Analysis}

As mentioned in the introduction, the present analysis consists of several interconnected ideas and vital steps which need to be explained consecutively with a reasonable degree of logical order as follows:

\subsection{KAM Spacetime Topology-The Pre-Space, Pre-Time and Pre-Matter}

Let us start by constructing a random Cantor set from the unit interval [7-10]. Proceeding in the usual way well known from the theory of fractals for the classical triadic Cantor set $\left(D_{H}=\ln 2 / \ln 3 \simeq 0.63\right)$ but adding uniform randomness to the construction procedure. That way we end up with a zero measure "thin" Cantor set of a topological dimension $D_{T}=0$ and a Hausdorff dimension very close to the classical deterministic set, namely $D_{H}=(\sqrt{5}-1) / 2=0.618033899$ [7-10]. Notice now that the gaps left from the iterative deletion of parts of the unit interval forms a second Cantor set. By contrast to the first "thin" Cantor set this one is a "fat" Cantor set with a positive measure equal to the length of the original unit interval because $L=1-0=1$. However in this case this Hausdorff dimension is not $\phi$ but obviously $1-\phi=\phi^{2}$ because for the original unit interval both the topological 
dimension and the Hausdorff dimension coincide and are equal to unity so that

$D_{H}(1)-D_{H}(0)=1-\phi=\phi^{2}$ as should be [7-10]. Noticing on the other hand that the thick Cantor set is made of totally empty gaps, it is clear that it is the epitome of the empty set which due to deductive dimensional and consistency reasons is assigned a topological dimension equal minus one, i.e. $D_{T}$ (empty) $=-1$ [22]. All the preceding result follows smoothly from Grothendieck $\mathrm{K}$-theoretical (i.e. the general theory of classes) conception of Penrose's fractal tiling universe [11-25] and Connes-von Neumann dimensional function [11-14] as well as E-infinity Cantorian spacetime bijection formula developed by the present author $[5,16]$. We showed previously how quantum physics could be formulated using a quantum set theory based on the preceding thin and thick Cantor sets [16]. These particles and waves arise naturally from a KAM-like fractal-Cantorian spacetime which is the ultimate source of matter and fields [18,22, 61]. Consequently this KAM spacetime picture is far more accurate than the ad hoc conventional picture of fractal spacetime [77-81] because we have only disjointed random sets interwoven together in an intricate manner but leaving only thin and fat Cantor sets to represent particles-zero sets and wave-empty sets respectively to form pre-space, pre-time and pre-matter to emerge from quantum spacetime geometry $[10,14,18,22$, 61]. In other words, quantum mechanical entanglement is what is behind the geometry of spacetime and vice versa $[20,21]$.

\subsection{The Zero Set Quantum Particle and the Empty Set Quantum Wave from a Generic Noncommutative Space and the Speed of Light as an Expectation Value}

In what follows we would like to make the KAM spacetime picture more specific. As hinted at in the previous section Penrose fractal tiling universe $[5,11,24]$ is the prototype par excellence of a noncommutative geometry reflecting the essence of K-theory [8] as well as E-infinity theory $[5,16,18,79]$ and its bijection formula which gives the Hausdorff dimension as a function of the zero set topological dimension $\phi$ and the topological dimension $\mathrm{n}[5,11]$. The corresponding dimension function is given by A. Connes in [11] as

$$
D=a+b ; a, b \quad \text { and }=\phi=\frac{\sqrt{5}-1}{2}
$$

For $a=0$ and $b=1$ one finds the bi-dimensions of the zero set, i.e.

$$
D_{o} \equiv\left(D_{T}, D_{H}\right) \equiv(0, \phi)
$$

where

$$
D_{T}=[D(a=0)+(b=1) \phi]=0+(1)(\phi)=\phi
$$

Similarly for the unit set we find [11-27]

$$
D_{H}=D[(a=1)+(b=0)(\phi)]=1+(0)(\phi)=1
$$

Subsequent dimensions are found using the Fibonacci prescription. For instance $D_{2}$ is found as

$$
D_{2}=(0+1)+(1+0) \phi=1+\phi=1 / \phi
$$

and so on. For the empty set $D-1$ the same procedure holds true but we need to mind the correct negative sign. That way one finds the Hausdorff dimension of the empty set to be [11-27]

$$
D_{-1}=(1-0)+(0-1) \phi=1-\phi=\phi^{2}
$$

The bijection formula on the other hand leads to the same result as mentioned at the beginning of this section however in a far simpler way because of the far more compact and economical notation. Thus from the bijection $[5,11,27]$

$$
d_{c}^{(n)}=(1 / \phi)^{n-1}
$$

we find the zero set by simply setting $n=0$

$$
d_{c}^{(0)}=(1 / \phi)^{0-1}=\phi
$$

while the empty set is found by setting $n=-1$

$$
d_{c}^{(-1)}=(1 / \phi)^{-1-1}=(1 / \phi)^{-2}=\phi^{2}
$$

Finally we mention on passing that the same result may be found using the gap labelling DIS method [12]. It is also obvious from the above that the empty set is the cobordism i.e. the surface of the zero set. In addition both the union and the intersection of the inverse dimensions of the zero and the empty set give rise to Hilbert-He's 4D fractal cube which is the core of our E-infinity space $[5,17]$. That means we are dealing with a KAM Cantorian space with indistinguishability condition $[13,74]$ :

$$
\begin{aligned}
& {\left[\frac{1}{D(\text { zero set })}\right] \cup\left[\frac{1}{D(\text { empty set })}\right]} \\
& =\left[\frac{1}{D(\text { zero set })}\right] \cap\left[\frac{1}{D(\text { empty set })}\right]
\end{aligned}
$$

Thus

$$
\begin{aligned}
\frac{1}{\phi}+\frac{1}{\phi^{2}} & =\left(\frac{1}{\phi}\right)\left(\frac{1}{\phi^{2}}\right)=\frac{1}{\phi^{3}} \\
& =4+\frac{1}{4+\frac{1}{4+\cdots}} \\
& =4+\phi^{3}
\end{aligned}
$$

which is probably the most recognizable formula of E-in- 
finity theory [5]. Another immensely important result of the von Neumann-Connes K-formula [11,18] and E-infinity bijection is that for $n=-\infty$ we have $d_{c}^{(-\infty)}=\phi^{\infty}=0$ [55]. That means we have an infinity of empty sets with an increasing degree of emptiness. This has physical consequences regarding the speed of light. For $d_{c}^{(-\infty)}=0$ the speed of light must be infinite. Consequently the observed constancy of the speed of light is nothing but the average speed of light in a fractal spacetime harbouring infinitely many empty sets [57]. One could acquire an intuitive feel for the problems by thinking about the speed of light in infinitely many layers of liquids with infinitely many different dimensions. That way the effective speed of light would be the average over infinitely many different velocities. The infinitely many dimensions of Cantorian spacetime play the same role of the liquids. In this sense one could say the constancy of the speed of light is the evidence that spacetime is a KAM fractal. Some experimental evidence for the reality of Cantorian spacetime is discussed by E. Goldfain in [62, 67].

\subsection{The Topological Invariants of KAM E-Infinity Space and Susslin Operation $[29,77]$}

Consider a space given by an infinite number of unions and intersections of elementary Cantor sets resembling a Susslin operation [29] of the form

$$
\mathscr{\delta}^{\infty} \equiv \bigcup_{0}^{\infty} \bigcap_{0}^{\infty} S^{(n)}
$$

so that

$$
D\left(\delta^{\infty}\right)=\sum_{n=0}^{\infty} n\left(d_{c}^{(n)}\right)^{n}
$$

where $n=0,1,2, \cdots$ and $d^{(n)}$ is the Hausdorff of the Cantor sets $S^{(n)}$. Since $d_{c}^{(0)} \leq 1$, the infinite series can be summed and one finds the topological dimension to be determined by the expectation value or a centre of gravity given by [5,15-24]

$$
\begin{aligned}
\langle n\rangle & =D\left(\mathcal{C}^{\infty}\right)=\frac{\sum_{0}^{\infty} n\left(d_{c}{ }^{(0)}\right)^{n}(n)}{\sum_{0}^{\infty} n\left(d_{c}^{(0)}\right)^{n}} \\
& =\frac{1+d_{c}{ }^{(0)}}{1-d_{c}^{(0)}}
\end{aligned}
$$

Noting that the average Hausdorff dimension is $[5$, 15-24]

$$
\begin{aligned}
\left\langle d_{c}\right\rangle & =\left(\sum_{0}^{\infty} d_{c}^{(n)}\right) / d_{c}^{(0)}=\left(\frac{1}{1-d_{c}^{(0)}}\right) / d_{c}^{(0)} \\
& =\frac{1}{d_{c}^{(0)}\left(1-d_{c}^{(0)}\right)}
\end{aligned}
$$

then by requiring space filling we see that we must have $\langle d c\rangle$ equal $\langle n\rangle$ and one finds the following condition [5,15-24]

$$
\frac{1+d_{c}^{(0)}}{1-d_{c}^{(0)}}=\frac{1}{d_{c}^{(0)}\left(1-d_{c}^{(0)}\right)}
$$

This leads to a quadratic equation in $d_{c}^{(0)}$ with two solutions of which the only positive one is $d_{c}{ }^{(0)}=(\sqrt{5}-1) / 2$ Inserting in $\langle n\rangle$ one finds that [5]

$$
\begin{aligned}
\langle n\rangle & =\left\langle d_{c}\right\rangle=(1+\phi) /(1-\phi)=1 /[\phi(1-\phi)] \\
& =1 / \phi^{3}=4+\phi^{3}=4.23603779
\end{aligned}
$$

In other words our E-infinity space is essentially the same space which we gained by considering the two Cantor sets constructed from the unit interval discussed earlier on [27]. We mention here without derivation that detailed analysis of E-infinity established a remarkable result, namely the equality of the curvature of the E-infinity manifold and its Euler characteristic which amounts to $26+k=26.18033989$ [55]. Note that $(26+k)^{2}$ is equal to the sum of all the 17 one and two Stein spaces [2].

\subsection{The Space E-Infinity $(E-\infty)$ as a Probability Space and Quantum Entanglement-The Immrizi Parameter and Unruh Temperature}

Let us derive a general expression for the probability that two different points in $8^{\infty}$ co-exist at the same location, i.e. being geometrically entangled. To do this we consider the probability of finding a single Cantor point in an isolated elementary Cantor set. The probability for that is obviously $P=\phi$ and for $\mathrm{n}$ point this is therefore given by the multiplication theorem to be $[3,16,27]$

$$
P_{1}=\phi^{n} \text {. }
$$

On the other hand the global probability and sometimes the contrafactual probability effect of finding a Cantor point in E-infinity is clearly the inverse of $\langle n\rangle$ namely $[3,16,27]$

$$
\begin{aligned}
P_{2} & =1 /\langle n\rangle=1 / 4.236067977 \\
& =\phi^{3}=0.23603779
\end{aligned}
$$

Consequently to find $\mathrm{n}$ point in Cantor set in E-infinity is the multiplication of the local probability $P_{1}=\phi^{n}$ with the global "contrafactual" probability $P_{2}=\phi^{3}$. This means the total probability is given by [3,17-19]

$$
P=P_{1} P_{2}=\phi^{n} \phi^{3}=\phi^{n+3} .
$$

Now we can distinguish various cases of $P$ with definite physical meaning corresponding to different numbers 
of particles $n$. The first is for $n=2$ which means the quantum entanglement of Hardy

$$
P(\text { Hardy })=\phi^{2+3}=\phi^{5} .
$$

This result was verified experimentally to a very high accuracy in various recognized laboratories [3,30-33]. The second value is for $n=3$ which gives us the famous Immirzi parameter of loop quantum gravity $[28,70]$

$$
P(\text { Immirzi })=\phi^{3+3}=\phi^{6} .
$$

The third result is that of the celebrated Unruh temperature for which we must set $n=1$ and consequently $[30,34]$

$$
P(\text { Unruh })=\phi^{1+3}=\phi^{4}
$$

In addition for $P=\phi^{5} / 2$ one finds the measurable ordinary energy density of the cosmos, i.e. the energy of the quantum particle $[2,34,35]$

$$
\begin{aligned}
\frac{E(\text { measured })}{E(\text { Einstein })} & =\frac{\left(\phi^{5} / 2\right) m c^{2}}{m c^{2}} \\
& =\phi^{5} / 2 \simeq 1 / 22
\end{aligned}
$$

Finally the microwave background radiation of the cosmos is found for $n=0$ to be related to geometrical self entanglement [36-38,67]

$$
P(\text { microwave })=\phi^{3}
$$

as discussed in more detail elsewhere [40-51]. Now we are actually in a position to answer Nobel Laureate G. 't Hooft's deep question "What are the building blocks of Nature?" The building blocks of nature and the building blocks of spacetime are the elementary random Cantor sets [64]. In addition we conjecture that the Unruh temperature is given by $\phi^{4}$ and that it is real, albeit a spacetime topological fractal effect [57].

\subsection{The Dark Energy of Quantum Kaluza-Klein Spacetime}

By raising the zero set modelling the quantum particle characterized by the bi-dimension $D_{o} \equiv(0, \phi)$ to literally the quintessence, i.e. the 5 dimensional Kaluza-Klein core of E-infinity, one easily arrives at an expression of energy density of the universe in complete agreement with cosmic measurement of COBE, WAMP and supernova burst analysis [34,35]. To show that we calculate the pseudo Hausdorff volume of Do which is a straight forward naive generalization of classical volume to $[26,27]$

$$
\operatorname{vol}^{(5)} D_{o}=\left(D_{H}\right)^{5}=\phi^{5} .
$$

Using Newton's kinetic energy as a template or simplistically speaking as a "Newtonian" rather than Hamil- tonian operator one finds $[26,27,61]$

$$
\begin{aligned}
E(0) & =\frac{1}{2} m(v \rightarrow c)^{2} \operatorname{vol}^{(5)} D_{o} \\
& =\left(\phi^{5} / 2\right) m c^{2}
\end{aligned}
$$

where $\mathrm{m}$ is the mass and $\mathrm{c}$ is the speed of light. Several vital points should be stressed at this stage. First while it is useful to distinguish sometime between mass, rest mass and relativistic mass the previous formula stresses that physical real mass does not change and that such concepts are only mathematical. The only "rest" quantity is the rest energy namely $E(0)$ itself. Second the speed of light $\mathrm{c}$ is quantitatively the same one we always used however its meaning here is different. The speed of light in E-infinity is variable and $\mathrm{c}$ is an expectation i.e. average value [10] as much as $4+\phi^{3}$ is the expectation for the formal topological dimension $d_{f}=\infty$ of E-infinity [5]. In this sense $E(0)$ represents a potential energy of the momentarily at resting measured quantum particle and we are entitled to ask now where is the kinetic energy? The answer of this question is as simple as it is surprisingly because it is the energy of the EinsteinBohm "ghost" quantum wave responsible for the propagation of the quantum particle [30,61]. To find the magnitude of this energy we calculate again a corresponding volume which turned out to be the dual value for $\phi^{5}$ namely an additive volume of the empty set $D-1$ in $D=$ $5[27,61]$

$$
\operatorname{vol}_{(5)}=(5)\left(\phi^{2}\right)
$$

where $D_{H}=\phi^{2}$ is the Hausdorff component of the empty set bi-dimension $D-1 \equiv\left(-1, \phi^{2}\right)$. The kinetic energy of the quantum wave in $\mathrm{D}=5$ is thus $[27,61]$

$$
E(D)=\left(5 \phi^{2} / 2\right) m c^{2} \text {. }
$$

The incredible fact which we should have noticed long ago but we did not is not only that $E(D)$ is the missing energy density of the cosmos $(95 \%$ of the total) but that $E(0)+E(D)=m c^{2}$ which is the classical expression found by Einstein using not solely mathematical deduction but also a quantum "leap" of "faith". In other words Einstein included quantum mechanical features in his famous formula although at the time of $E=m c^{2}$ quantum mechanics was not yet invented and that later on when quantum mechanics was around Einstein did not believe in it because of the spooky action at distance of quantum entanglement. Ironically the energy expression $E(0)$ consists of two parts, namely $\mathrm{mc}^{2}$ multiplied with $\phi^{5} / 2$ where $\phi^{5}$ is the Hardy probability of quantum entanglement of two quantum particles so that $\phi^{5} / 2$ accounts for the effect of quantum entanglement of the one particle energy expression. There is an even simpler interpretation of $E(0)$ and consequently 
$E(D)=1-E(0)$ when involving the mathematics and physics of Nambu-Veneziano's old bosonic string theory of strong interaction. According to this theory the spacetime dimensions needed are not 4 but 26 . Consequently if we understand $E=m c^{2}$ as a theory for $D=4$ then according to the $D=26$ theory we have ignored the effect of the 26-4 = 22 "compactified" dimensions of spacetime [27,57]. Since $E$ is an Eigenvalue, then by Rayleigh theorem we should reduce by division $E=m c^{2}$ which means Weyl-Nottale scale [14] it using a scaling exponent $\lambda=1 / 22$ so that we find

$$
E(0)=m c^{2} / 22 .
$$

Noting that the exact value is

$$
E(0)=\left(\phi^{5} / 2\right) m c^{2}=\left(\frac{1}{22.18033989}\right) m c^{2}
$$

We see that $E(0)=m c^{2} / 22$ is an excellent integer approximation [27,28,34,35]. Similarly the dark energy density of the quantum wave

$$
\begin{aligned}
E(D) & =\left(5 \phi^{5} / 2\right) m c^{2} \\
& =(21.18033989 / 22.18033989)\left(m c^{2}\right)
\end{aligned}
$$

could be approximated to [27]

$$
E(D)=m c^{2}(21 / 22) \text {. }
$$

The preceding two results are probably the most important achievements of many research efforts of many scientists [1-51] rather than a single person and could be truly said to stand on the shoulders of giants [53]. From this privileged position we can now answer a second deep question by G. Ord [63], namely "What is the wave function?" From the above it is clear that it is the empty set or the surface of the zero set quantum particle. In addition the wave function is the source of dark energy $[61,63]$. We conjecture or more accurately speculate using educated scientific guessing that some topological defects [72] like texture or even instantons may have an intrinsic topological dimension equal to minus one making them de facto empty sets and thus a source of negative dark energy and consequently negative gravity [2]. On the other hand we could give a more conservative interpretation by regarding $\mathrm{E}(\mathrm{D})$ as simply the energy vacuum surrounding a mini black hole, i.e. a pre-quantum particle with $\phi^{5} / 2$ being information entropy while $1-\left(\phi^{5} / 2\right)=5 \phi^{2} / 2$ is the lack of information complimentary entropy. This mini black hole picture could be seen as suggesting that dark energy is produced from Hawking-Beckenstein entropy leading to negative energy fluctuation at the mini black hole horizon as will be discussed next from a complimentary geometrical view point.

\subsection{Accelerated Cosmic Expansion, Anticlastic Curvature and Hawking's Negative Vacuum Fluctuation Energy Fluctuation-Negative Van der Waals Processes [76]}

To make a long story short, the surprising discovery of the accelerating rather than decelerating expansion of the universe is argued here to be due to the well known geometrical effect of anticlastic, i.e. negative curvature of the spacetime manifolds of the cosmos. In turns this anticlastic curvature accumulates at the edge of the world to a maximum as can be seen from the analogy with a long elastic thin walled cylinder squeezed at the middle as shown in Figure 14 of Ref. [2] as well as Ref. [83] Figure 2. The situation is thus analogous to that of the negative energy of vacuum fluctuation found by S. Hawking to exist at the horizon of a black hole. In other words when we look at the two dimensional projection of a ramified i.e. compactified Klein modular curve or a fractal Penrose universe [65], then at the edges being in the hyperbolic plane at infinity surrounded by a Cantorian circle we have anticlastic curvature, i.e. negative curvature producing negative dark energy causing negative gravity pushing the universe apart rather than pulling it together. The preceding geometrical picture could be translated to a topological picture by reasoning that in simplistic terms, while the visible 4 dimensions of our space produce positive curvature and ordinary gravity, the $26-4=22$ compactified bosonic string dimensions are responsible for dark energy, i.e. negative curvature and consequently negative gravity which is the force behind the measured increased acceleration of cosmic expansion at the edge of the holographic Klein-Penrose universe [65]. The situation can be explained quantitatively by taking the ratio between Heterotic string theory 504 states and the 528 states of maximally symmetric spaces such as Witten's M-theory [2-4,10,27,28]. There is also a very instructive analogy to between gravity and Van der Waals fluctuation which was proposed by the author [75] and which could be extended to negative gravity and negative Van der Waals fluctuation [76].

\subsection{Scaling the Planck Scale-The Connection between Wormholes (Einstein-Rosen Bridges) and Quantum Entanglement (Einstein-Podolsky-Rosen Spooky Action at Distance)}

The Planck energy $E_{P} \simeq 10^{19} \mathrm{Gev}$ and not dissimilarly the Planck length $\ell_{P} \cong 10^{-33} \mathrm{~cm}$ are quite esoteric scales difficult to visualize and totally outside present or near future experimental capacities if at all [30]. Never the less by going back to our initial unit interval universe and noting the unexpected result arising from Hardy's entanglement $P($ Hardy $)=\phi^{5}$ and related conclusions 
regarding Sigalotti's critical "topological" velocity of light $c=\phi$ and the isomorphic length of a super symmetric compactified hyperbolic Penrose universe [65] with a Hawking black hole circular horizon given by [10, 61]:

$$
\begin{aligned}
\ell & =\left(4+\phi^{3}\right)\left(5+\phi^{3}\right) / 2=(22+k) / 2 \\
& =11+(k / 2)=11+\phi^{5} \\
& =11+\frac{1}{11+\frac{1}{11+\cdots}}
\end{aligned}
$$

we realize the following vital topological facts which amount to a realization of D. Gross' idea of scaling the Planck scale [10]:

1) The topological Planck energy is nothing but Hardy's quantum entanglement, i.e. the quantum glue which sticks the patches of spacetime together

$$
E_{p}=P(H)=\phi^{5}
$$

2) The topological expectation value of the speed of light in a multi-fractal spacetime medium is

$$
c=\phi \text {. }
$$

3) The topological Planck length is equal to the dimension of a fractal M-theory

$$
\ell_{P}=11+\phi^{5}
$$

since $11+\phi^{5}$ is equal to $1 / \phi^{5}$ where $\phi^{5}$ is the topological Plank energy $E_{P}$ we see that E-infinity space is infinitely multi-connected and that all these fractal gaps and voids in their space are essentially wormholes [6] with length equal to an isomorphic length $\ell_{P}=11+\phi^{5}$ of a super symmetric Penrose tiling universe which constitutes Einstein-Rosen bridges [6,30]. It is ironic as well as surreal to see that the ultimate resolution of the Einstein-Podolsky-Rosen spooky action at distance existed always in the Einstein-Rosen bridges and consequently in quantum entanglement which Einstein as well as Schrödinger rejected so vehemently [52].

\subsection{Ordinary and Dark Energy from Rindler Space}

A truly brief derivation of dark energy which may also highlight strong evidence that the Rindler-Unruh effects [82] are real and similar to Hawking's negative energy fluctuation at a black hole horizon [82] could run as follows:

The topological ordinary energy density is simply half the Unruh topological mass $\phi^{3}$ multiplied with the square of the topological speed of light $\phi^{2}$ [2]. That means it is exactly equal to the area of the fractal version of the hyperbolic Rindler triangle (see Ref. [2], Figures 1.2 to 1.6$]$ when we realize that fractal geometry has no ordinary lines but borders between different KAM space fractal parts. Since events in this hyperbolic fractal front triangle are correlated, i.e. entangled, we half obtained half of Hardy's $P(H)=\phi^{5}$ quantum entanglement probability and the final result for ordinary energy density is $E(O)=\left(\phi^{5} / 2\right) m c^{2}$. The topological dark energy density on the other hand is equal to half of Kaluza-Klein's topological mass 5 multiplied with the square of the topological speed of light $\phi^{2}$ [2]. That means we have $5 \phi^{2} / 2$ which is exactly equal to the area behind the hyperbolic triangle which completes it to the famous Lorentzian invariant Rindler triangular wedge [82]. The final value of the dark energy is thus $E(D)=\left(5 \phi^{2} / 2\right) m c^{2}$. This dark energy is uncorrelated and cannot be observed by the Rindler observer [82].

\section{Results and Discussion}

At the beginning there was the word topology constituting the blueprint for existence. However for the Pythagoreans it was the number which may be golden mean number system in which the topological properties of elementary Cantor sets are expressed so that we can do calculations with them and draw general conclusions $[4,17,45]$. The author hopes that the present work makes it clear that we can deal with infinity without processing an infinite number of information $[17,54,56]$. In fact $\phi$ written in decimal expansion $0.618033989 \ldots$ is infinite. However written as $(\sqrt{5}-1) / 2$ we can easily work out that $\phi^{2}=(5+1-2 \sqrt{5}) / 2=3-\sqrt{5}$ and that $\phi+\phi^{2}=1$ [45]. In any event we showed here beyond any reasonable doubt that $E=m c^{2}$ consists of two parts, $E(0)=m c^{2} / 22$. which is the position or potential energy of a quantum particle or equivalently a five dimensional "quintic" zero set at rest and $E(D)=m c^{2}(21 / 22)$ which is the absolute value of the negative kinetic energy of the quantum wave or equivalently the moving "quintic" empty set of a quasi De-Sitter five dimension space $[2,61]$. Together $E(0)+E(D)=m c^{2}$ give Einstein's iconic formula for the maximal total energy of a classical particle $[4,26,61]$. It is important to note the $E(0)$ density is only $4.5 \%$ of the total expected energy density of the universe in agreement with the most recent sophisticated accurate cosmological measurements of the three physics Nobel Prize winners of 2011 [34,35,61]. On the other hand present measurement technologies do not permit direct measurement of $E(D)$ which constitutes $95.5 \%$ of the total expected energy because of the well documented but ill understood quantum wave collapse at measurement $[10,20,33]$. For this reason $E(D)$ is dubbed dark energy and baring new developments in wave collapse free non-demolition measurement instruments $[2,4,10,20]$, dark it will remain $[30,34,35]$. Negative dark energy is linked here to the anticlastic curvature at the 
edge of the a Rindler Wedge-like universe and is therefore a cousin of Hawking's negative energy fluctuation of the vacuum at the horizon of a toy black hole which constitutes a circular edge of the hyperbolic compactified edge lying at infinity of a Klein-Penrose holographic universe $[2,61,65]$. It is this negative gravity force which is responsible for the unexpected measured accelerated expansion of the cosmos which is the only indication that repulsive gravity due to negative dark energy must really be there $[10,34,35]$. Technically speaking we could not have obtained accurate quantitative results for the preceding effect if it would not have been for L. Hardy's magnificent quantum entanglement exact solutions for two particles [31-33] as well as the work of Sigalotti [10] leading to the topological speed of light $c=\phi$. In addition the general theory of noncommutative space of A. Connes and the realization that Penrose universe is a K-theoretical explicit realization of a generic noncommutative space played a pivotal role in reaching our conclusion [2].

\section{Conclusion}

Finally the inspiring idea of D. Gross to scale the Planck scale [1] gave an overall intuitive justification for $E_{P}=\phi^{5}$ and the fractal M-theory dimension $\ell_{P}=11+\phi^{5}$ to realize that E-infinity fractal geometry is the geometry of quantum entanglement linking Einstein-Rosen bridges with quantum entanglement, zero measure geometry as well as Mielke-El Naschie-Elokaby worm holes and Magueijo-Smolin's ingenious theory of varying speed of light [44]. The same is true for Nash Embedding of Witten's M-Theory [83]. In fact from the fundamental work of Gutzwiller, the Russian School of Quantum Chaos led by Cherikov and the pioneering work of Berry, Casati and others on KAM [80-83], it is clear that quantum entanglement shapes quantum spacetime and glues it together giving rise to classical space and time [3]. The entire analysis and result obtained have testified once more to Einstein's dictum "Raffiniert is der Herr Gott aber boshaft ist er nich". Einstein added later that "Die Natur verbirgt ihre Geheimnisse durch die Erhabenheit ihres wesen, aber nicht durch List" [52]. In fact nature is simple to a degree that at least initially defies human expectation, in order not to say human comprehension. However, as H.R.H. the Prince of Wales, T. Juniper and I. Shelly emphasized in their book [53], harmony is the way to comprehension and nothing could be more harmonious than a golden ratio based number system which we used in E-infinity theory $[17,53,2]$. In E-infinity the most irrational number $\phi$ with infinite decimal expansion $\phi=(\sqrt{5}-1) / 2=0.618033989$ is taken as basic and integers as constructed from $\phi+\phi^{2}=1$ and so on. Similarly rationals are given by

$\left(\phi+\phi^{2}\right) /\left(\phi+\phi+\phi^{2}+\phi^{2}\right)=1 / 2$. We note on passing that the golden mean is fundamental in KAM theorem $[3,8,80]$. In that way we tame infinity and extend what D. Hilbert called Cantor's paradise to physics $[17,54]$. The author for one firmly believes in the preceding concept of the role of infinity in physics and the incredibly deep work of Hugh Wooden [56], which is bigger than mere mathematical tradition which came from G. Cantor, a monastery in Greece and the Moscow School of Mathematics founded by Egorov and Luzin [77].

\section{REFERENCES}

[1] D. J. Gross, "Can We Scale the Planck Scale?" Physics Today, Vol. 42, No. 6, 1989, pp. 9-11. http://dx.doi.org/10.1063/1.2811040

[2] M. S. El Naschie and A. Helal, "Dark Energy Explained via the Hawking-Hartle Quantum Wave and the Topology of Cosmic Crystallography," Int. J. Astron. \& Astrophys, Vol. 3, No. 3, 2013, p. 318.

[3] M. S. El Naschie, "Quantum Entanglement as a Consequence of a Cantorian Micro Spacetime Geometry," Journal of Quantum Information Science, Vol. 1, No. 2, 2011, pp. 50-53. http://dx.doi.org/10.4236/jqis.2011.12007

[4] M. S. El Naschie, "A Resolution of Cosmic Dark Energy via a Quantum Entanglement Relativity Theory,” Journal of Quantum Information Science, Vol. 3, No. 1, 2013, pp. 23-26. http://dx.doi.org/10.4236/jqis.2013.31006

[5] M. S. El Naschie, "A Review of $E$ Infinity Theory and the Mass Spectrum of High Energy Particle Physics," Chaos, Solitons \& Fractals, Vol. 19, No. 1, 2004, pp. 209-236. http://dx.doi.org/10.1016/S0960-0779(03)00278-9

[6] A. Elokaby, "Knot Wormholes and the Dimensional Invariant of Exceptional Lie Groups and Stein Space Hierarchies," Chaos, Solitons \& Fractals, Vol. 41, No. 4, 2009, pp. 1616-1618. http://dx.doi.org/10.1016/j.chaos.2008.07.003

[7] P. S. Addison, "Fractals and Chaos. An Illustrated Course," IOP, Bristol, 1997. http://dx.doi.org/10.1887/0750304006

[8] F. Diacu and P. Holmes, "Celestial Encounters-The Origins of Chaos and Stability," Princeton University Press, Princeton, 1996.

[9] M. S. El Naschie, “A Fractal Menger Sponge Space-Time Proposal to Reconcile Measurements and Theoretical Predictions of Cosmic Dark Energy," International Journal of Modern Nonlinear Theory and Application, Vol. 2, No. 2, 2013, pp. 107-121. http://dx.doi.org/10.4236/ijmnta.2013.22014

[10] M. S. El Naschie, "Quantum Entanglement: Where Dark Energy and Negative Gravity plus Accelerated Expansion of the Universe Comes from," Journal of Quantum Information Science, Vol. 3, No. 2, 2013, pp. 57-77. http://dx.doi.org/10.4236/jqis.2013.32011

[11] A. Connes, "Noncommutative Geometry," Academic Press, New York, 1994.

[12] J. Bellissard, "Chapter 12: Gap Labeling Theorems for Schrödinger Operators,” In: M. Waldschmidt, P. Monsa, J. 
Luck and C. Itzykon, Eds., From Number Theory to Physics, Springer, Berlin, 1992.

[13] G. Landi, "An Introduction to Noncommutative Space and Their Geometries," Springer, Berlin, 1997.

[14] L. Marek-Crnjac, M. S. El Naschie and J.-H. He, "Chaotic Fractals at the Root of Relativistic Quantum Physics and Cosmology," International Journal of Modern Nonlinear Theory and Application, Vol. 2, No. 1A, 2013, pp. 78-88. http://dx.doi.org/10.4236/ijmnta.2013.21A010

[15] M. S. El Naschie and J.-H. He, "Fractal Hilbert Space as the Geometry of Quantum Mechanical Entanglement," Fractal Space-Time and Non-Commutative Geometry in High Energy Physics, Vol. 2, No. 1, 2012, p. 41.

[16] M. S. El Naschie, "Towards a General Transfinite Set Theory for Quantum Mechanics," Fractal Space-Time and Non-Commutative Geometry in High Energy Physics, Vol. 2, No. 2, 2012, pp. 135-142.

[17] M. S. El Naschie, J.-H. He, S. Nada, L. Marek-Crnjac and M. Atef Helal, "Golden Mean Computer for High Energy Physics," Fractal Space-Time and Non-Commutative Geometry in High Energy Physics, Vol. 2, No. 2, 2012, pp. 80-92.

[18] J.-H. He and M. S. El Naschie, "On the Monadic Nature of Quantum Gravity as a Highly Structured Golden Ring Spaces and Spectra," Fractal Space-Time and NonCommutative Geometry in High Energy Physics, Vol. 2, No. 2, 2012, p. 94.

[19] M. S. El Naschie and J.-H. He, "The Fractal Geometry of Quantum Mechanics Revealed," Fractal Space-Time and Non-Commutative Geometry in High Energy Physics, Vol. 1, No. 1, 2011, p. 3.

[20] M. S. El Naschie and S. Olsen, "When Zero Is Equal to One: A Set Theoretical Resolution of Quantum Paradoxes," Fractal Space-Time and Non-Commutative Geometry in High Energy Physics, Vol. 1, No. 1, 2011, p. 11.

[21] M. S. El Naschie and O. E. Rossler, "The Zero Measure Fractal Ghost inside Quantum Mechanics," Fractal SpaceTime and Non-Commutative Geometry in High Energy Physics, Vol. 2, No. 1, 2012, p. 56.

[22] J.-H. He, T. Zhong, L. Xu, L. Marek-Crnjac, S. Nada and M. Atef Helal, "The Importance of the Empty Set and Noncommutative Geometry in Underpinning the Foundations of Quantum Physics," Nonlinear Science Letters B, Vol. 1, No. 1, 2011, p. 15.

[23] M. S. El Naschie, "Quantum Collapse of Wave Interference Pattern in the Two-slit Experiment: A Set Theoretical Resolution," Nonlinear Science Letters B, Vol. 2, No. 1, 2011, p. 1.

[24] M. S. El Naschie, L. Marek-Crnjac, J.-H. He and M. Atef Helal, "Computing the Missing Dark Energy of a Clopen Universe which Is Its Own Multiverse in Addition to Being Both Flat and Curved," Fractal Space-Time and NonCommutative Geometry in High Energy Physics, Vol. 3, No. 1, 2013, p. 3.

[25] T. Zhong and J.-H. He, "El Naschie's Resolution of the Mystery of Missing Dark Energy of the Cosmos via Quantum Field Theory in Curved Spacetime," Fractal
Space-Time and Non-Commutative Geometry in High Energy Physics, Vol. 3, No. 1, 2013, p. 46.

[26] M. S. El Naschie, "Dark Energy from Kaluza-Klein Spacetime and Noether's Theorem via Lagrangian Multiplier Method," Journal of Modern Physics, Vol. 4, No. 6, 2013, pp. 757-760.

http://dx.doi.org/10.4236/jmp.2013.46103

[27] M. S. El Naschie, "Topological-Geometrical and Physical Interpretation of the Dark Energy of the Cosmos as a "Halo" Energy of the Schrödinger Quantum Wave," Journal of Modern Physics, Vol. 4, No. 5, 2013, pp. 591-596. http://dx.doi.org/10.4236/jmp.2013.45084

[28] M. S. El Naschie, "The Quantum Gravity Immirzi Parameter-A General Physical and Topological Interpretation," Gravitation and Cosmology, Vol. 19, No. 3, 2013, pp. 151-155. http://dx.doi.org/10.1134/S0202289313030031

[29] S. Krantz and H. Parks, "Geometric Integration Theory," Birkhäuser, Boston, 2008. http://dx.doi.org/10.1007/978-0-8176-4679-0

[30] R. Penrose, "The Road to Reality," Johnathan Cape, London, 2004.

[31] L. Hardy, "Nonlocality for Two Particles without Inequalities for Almost All Entangled States," Physical Review Letters, Vol. 71, No. 11, 1993, p. 1665-1668. http://dx.doi.org/10.1103/PhysRevLett.71.1665

[32] P. Kwiat and L. Hardy, "The Mystery of the Quantum Cakes," American Journal of Physics, Vol. 68, No. 1, 2000, p. 33. http://dx.doi.org/10.1119/1.19369

[33] N. D. Mermin, "Quantum Mysteries Refined," American Journal of Physics, Vol. 62, No. 10, 1994, p. 880. http://dx.doi.org/10.1119/1.17733

[34] E. J. Copeland, M. Sami and S. Tsujikawa, "Dynamics of Dark Energy," arXiv: hep-th/ 0603057V3, 2006. http://arxiv.org/pdf/hep-th/0603057

[35] S. Perlmutter, et al., "Supernova Cosmology Project Collaboration," The Astrophysical Journal, Vol. 517, No. 2, 1999, p. 565. http://dx.doi.org/10.1086/307221

[36] M. S. El Naschie, "A Remark on the Cosmic Microwave Background Radiation and the Hausdorff Dimension of Spacetime," Chaos, Solitons \& Fractals, Vol. 10, No. 11, 1999, pp. 1807-1811. http://dx.doi.org/10.1016/S0960-0779(99)00008-9

[37] M. S. El Naschie, "Derivation of the Threshold and Absolute Temperature $\mathrm{Tc}=273.16 \mathrm{~K}$ from the Topology of Quantum Space-Time," Chaos, Solitons \& Fractals, Vol. 14, No. 7, 2002, pp. 1117-1120.

http://dx.doi.org/10.1016/S0960-0779(02)00053-X

[38] M. S. El Naschie, "Cobe Satellite Measurement, Hyperspheres, Superstrings and the Dimension of Spacetime," Chaos, Solitons \& Fractals, Vol. 9, No. 8, 1998, pp. 14451471. http://dx.doi.org/10.1016/S0960-0779(98)00120-9

[39] M. S. El Naschie, "Fuzzy Multi-Instanton Knots in the Fabric of Space-Time and Dirac's Vacuum Fluctuation," Chaos, Solitons \& Fractals, Vol. 38, No. 5, 2008, pp. 12601268. http://dx.doi.org/10.1016/j.chaos.2008.07.010

[40] M. S. El Naschie, "Fractal Black Holes and Information," 
Chaos, Solitons \& Fractals, Vol. 29, No. 1, 2006, pp. 23-35. http://dx.doi.org/10.1016/j.chaos.2005.11.079

[41] M. S. El Naschie, "Knots and Exceptional Lie Groups as Building Blocks of High Energy Particle Physics," Chaos, Solitons \& Fractals, Vol. 41, No. 4, 2009, pp. 1799-1803. http://dx.doi.org/10.1016/j.chaos.2008.07.025

[42] M. S. El Naschie, "Arguments for the Compactness and Multiple Connectivity of Our Cosmic Spacetime," Chaos, Solitons \& Fractals, Vol. 41, No. 5, 2009, pp. 2787-2789. http://dx.doi.org/10.1016/j.chaos.2008.10.011

[43] M. S. El Naschie, "Transfinite Neoimpressionistic Reality of Quantum Spacetime," New Advances in Physics, Vol. 1, No. 2, 2007, p. 111.

[44] J. Mageuijo and L. Smolin, "Lorentz Invariance with an Invariant Energy Scale," arXiv: hep-th/0112090V2, 2001. http://arxiv.org/pdf/hep-th/0112090

[45] T. Zhong, "From the Numerics of Dynamics to the Dynamics of Numerics and Visa Versa in High Energy Particle Physics," Chaos, Solitons \& Fractals, Vol. 42, No. 3, 2009, pp. 1780-1783.

http://dx.doi.org/10.1016/j.chaos.2009.03.079

[46] M. S. El Naschie, "Determining the Temperature of the Microwave Background Radiation from the Topology and Geometry of Spacetime," Chaos, Solitons \& Fractals, Vol. 14, No. 7, 2002, pp. 1121-1126. http://dx.doi.org/10.1016/S0960-0779(02)00172-8

[47] M. S. El Naschie, "On the Vital Role Played by the Electron-Volt Units System in High Energy Physics and Mach's Principle of 'Denkökonomie',' Chaos, Solitons \& Fractals, Vol. 28, No. 5, 2006, pp. 1366-1371. http://dx.doi.org/10.1016/j.chaos.2005.11.001

[48] M. S. El Naschie, "Holographic Dimensional Reduction: Center Manifold Theorem and E-Infinity," Chaos, Solitons \& Fractals, Vol. 29, No. 4, 2006, pp. 816-822. http://dx.doi.org/10.1016/j.chaos.2006.01.013

[49] M. S. El Naschie, "Modular Groups in Cantorian $E^{(\infty)}$ High-Energy Physics," Chaos, Solitons \& Fractals, Vol. 16, No. 2, 2003, pp. 353-366. http://dx.doi.org/10.1016/S0960-0779(02)00440-X

[50] M. S. El Naschie, "Quantum Loops, Wild Topology and Fat Cantor Sets in Transfinite High-Energy Physics," Chaos, Solitons \& Fractals, Vol. 13, No. 5, 2002, pp. 1167-1174. http://dx.doi.org/10.1016/S0960-0779(01)00210-7

[51] M. S. El Naschie, "Wild Topology, Hyperbolic Geometry and Fusion Algebra of High Energy Particle Physics," Chaos, Solitons \& Fractals, Vol. 13 No. 9, 2002, pp. 19351945. http://dx.doi.org/10.1016/S0960-0779(01)00242-9

[52] A. Pais, "Subtle Is the Lord: The Science and Life of Albert Einstein," Oxford University Press, Oxford, 1982.

[53] J. H. He and M. S. El Naschie, "On the Shoulders of Giants," Fractal Space-Time and Non-Commutative Geometry in High Energy Physics, Vol. 3, No. 1, 2013, p. 59.

[54] A. Gefter, "Mind-Bending Mathematics: Why Infinity Has to Go," New Scientist, Vol. 219, No. 2930, 2013, pp. 32-35. http://dx.doi.org/10.1016/S0262-4079(13)62043-6

[55] M. S. El Naschie, "The Theory of Cantorian Spacetime and High Energy Particle Physics (An Informal Review)," Chaos, Solitons \& Fractals, Vol. 41, No. 5, 2009, pp. 26352646. http://dx.doi.org/10.1016/j.chaos.2008.09.059

[56] R. Elwes, "Ultimate Logic," New Scientist, Vol. 211, No. 2823, 2011, pp. 30-33. http://dx.doi.org/10.1016/S0262-4079(11)61838-1

[57] J. H. He and L. Marek-Crnjac, "Mohamed El Naschie's Revision of Albert Einstein's $E=m_{0} c^{2}$ : A Definite Resolution of the Mystery of the Missing Dark Energy of the Cosmos," International Journal of Modern Nonlinear Theory and Application, Vol. 2, No. 1, 2013, pp. 55-59. http://dx.doi.org/10.4236/ijmnta.2013.21006

[58] M. S. El Naschie, "Superstrings, Knots, and Noncommutative Geometry in $E^{(\infty)}$ Space," International Journal of Theoretical Physics, Vol. 37, No. 12, 1998, pp. 2935-2951. http://dx.doi.org/10.1023/A:1026679628582

[59] S. Chattopadhyay and A. Pasqua, "Various Aspects of Interacting Modified Holographic Ricci Dark Energy," Indian Journal of Physics, Vol. 87, No. 10, 2013, pp. 10531057.

[60] S. Kalita, H. L. Duorah and K. Duorah, "Late Time Cosmic Acceleration of a Flat Matter Dominated Universe with Constant Vacuum Energy," Indian Journal of Physics, Vol. 84, No. 6, 2013, p. 629.

[61] M. S. El Naschie, "The Missing Dark Energy of the Cosmos from Light Cone Topological Velocity and Scaling of the Planck Scale," Open Journal of Microphysics, Vol. 3, No. 3, 2013, pp. 64-70. http://dx.doi.org/10.4236/ojm.2013.33012

[62] E. Goldfain, "On a Possible Evidence for Cantorian SpaceTime in Cosmic Ray Astrophysics," Chaos, Solitons \& Fractals, Vol. 20, No. 3, 2004, pp. 427-435. http://dx.doi.org/10.1016/j.chaos.2003.10.012

[63] G. N. Ord, "Quantum Mechanics in a Two-Dimensional Spacetime: What Is a Wave Function?" Annals of Physics, Vol. 324, 209, 2004, p. 1211.

[64] M. S. El Naschie, "“t Hooft Ultimate Building Blocks and Space-Time as an Infinite Dimensional Set of Transfinite Discrete Points," Chaos, Solitons \& Fractals, Vol. 25, No. 3, 2005, pp. 521-524. http://dx.doi.org/10.1016/j.chaos.2005.01.022

[65] A. Elokaby, "On the Deep Connection between Instantons and String States Encoder in Klein's Modular Space," Chaos, Solitons \& Fractals, Vol. 42, No. 1, 2009, pp. 303305. http://dx.doi.org/10.1016/j.chaos.2008.12.001

[66] G. Iovane and S. I. Nada, "Strange Non-Dissipative and Non-Chaotic Attractors and Palmer's Deterministic Quantum Mechanics," Chaos, Solitons \& Fractals, Vol. 42, No. 1, 2009, pp. 641-642. http://dx.doi.org/10.1016/j.chaos.2008.11.024

[67] J. H. He, E. Goldfain, L. D. Sigalotti and A. Mejias, "Beyond the 2006 Physics Nobel Prize for COBE," China Culture \& Scientific Publishing, Shanghai, 2006.

[68] M. S. El Naschie, "The Hyperbolic Extension of SigalottiHendi-Sharifzadeh's Golden Triangle of Special Theory of Relativity and the Nature of Dark Energy," Journal of Modern Physics, Vol. 4, No. 3, 2013, pp. 354-356. http://dx.doi.org/10.4236/jmp.2013.43049 
[69] D. R. Finkelstein, “Quantum Relativity," Springer, Berlin, 1996. http://dx.doi.org/10.1007/978-3-642-60936-7

[70] C. Rovelli, "Quantum Gravity," Cambridge Press, Cambridge, 2004. http://dx.doi.org/10.1017/CBO9780511755804

[71] Y. Gnedin, A. Grib and V. Matepanenko, "Quantum Gravity," Proceedings of the Third Alexander Friedmann Int. Seminar on Gravitation and Cosmology, Friedmann Lab. Pub., St. Petersberg, 1995.

[72] A. Vileukin and E. Shellard, "Cosmic Strings and Other Topological Defects," Cambridge University Press, Cambridge, 2001.

[73] M. S. El Naschie, "On the Uncertainty of Cantorian Geometry and the Two-Slit Experiment," Chaos, Solitons \& Fractals, Vol. 9, No. 3, 1998, pp. 517-529. http://dx.doi.org/10.1016/S0960-0779(97)00150-1

[74] M. A. Helal, L. Marek-Crnjac and J.-H. He, "The Three Page Guide to the Most Important Results of M. S. El Naschie's Research in E-Infinity Quantum Physics and Cosmology," Open Journal of Microphysics, Vol. 3, No. 4, 2013.

[75] M. S. El Naschie, "A Note on Quantum Gravity and Cantorian Spacetime," Chaos, Solitons \& Fractals, Vol. 8, No. 1, 1997, pp. 131-133.

http://dx.doi.org/10.1016/S0960-0779(96)00128-2
[76] V. Adrian Parsegian, "Van der Waals Forces," Cambridge University Press, Cambridge, 2006.

[77] L. Graham and J. Kantor, "Naming Infinity," Harvard University Press, Cambridge, 2009.

[78] M. Persinger and C. Lavellee, "Theoretical and Experimental Evidence of Macroscopic Entanglement between Human Brain Activity and Photon Emission," Journal of Consciousness Exploration \& Research, Vol. 1, No. 7, 2010, pp. 785-807.

[79] S. Vrobel, "Fractal Time," World Scientific, Singapore, 2011.

[80] G. Casati, I. Guarneri and U. Smilansky, "Caos Quantico," Proceedings of the International School of Physics "Enrico Fermi", Amsterdam, 1993.

[81] L. Nottale, "Scale Relativity and Fractal Spacetime," Imperial College Press, London, 2011.

[82] L. Susskind and J. Lidesay, "Black Holes, Information and the String Theory Revolution," World Scientific, Singapore, 2010.

[83] M. S. El Naschie, "Nash Embedding of Witten's M-Theory and the Hawking-Hartle Quantum Wave of Dark Energy," Journal of Modern Physics, Vol. 4, No. 10, 2013, pp. 1417 1428. 